\title{
INTERPOLATION AND INEQUALITIES FOR FUNCTIONS OF EXPONENTIAL TYPE: THE ARENS IRREGULARITY OF AN EXTREMAL ALGEBRA
}

\author{
by M. J. CRABB and C. M. McGREGOR
}

(Received 10 April, 1992)

For any compact convex set $K \subset \mathbb{C}$ there is a unital Banach algebra $E a(K)$ generated by an element $h$ in which every polynomial in $h$ attains its maximum norm over all Banach algebras subject to the numerical range $V(h)$ being contained in $K,[1]$. In the case of $K$ a line segment in $\mathbb{R}$, we show here that $E a(K)$ does not have Arens regular multiplication. We also show that ideas about $E a(K)$ give simple proofs of, and extend, two inequalities of $C$. Frappier [4].

For $K=[-\tau, \tau], \tau>0$, the generator $h$ is Hermitian; equivalently, $\left\|e^{i t h}\right\|=1(t \in \mathbb{R})$, with $V(h)=\operatorname{Sp}(h)=[-\tau, \tau]$. For the inequalities we use the fact that the operator $D$ of differentiation on $B_{r}$, the space of entire functions $f$ such that $|f(z)| e^{-\tau|l m z|}$ is bounded for $z \in \mathbb{C}$, is $i$ times a Hermitian, where we give $f \in B_{\tau}$ the norm $\|f\|=\sup \{|f(x)|: x \in \mathbb{R}\}$. This is all we require here; in fact with $h=-i D$ this gives a realization of $E a[-\tau, \tau],[2]$.

We may assume, replacing $h$ by $\alpha h+\beta$ for suitable $\alpha, \beta \in \mathbb{C}$, that $K=[-2 \pi, 2 \pi]$, so that $h$ is Hermitian: that is, $\left\|e^{i t h}\right\|=1(t \in \mathbb{R})$. As in [2], any entire function $f$ such that $|f(z)| \exp (-2 \pi|\operatorname{Im} z|)$ is bounded for $z \in \mathbb{C}$ gives a functional $\phi \in E a(K)^{\prime}$, by $\phi\left(e^{i z h}\right)=$ $f(z)(z \in \mathbb{C})$. By [5] a Banach algebra $A$ is Arens irregular if, for some bounded sequences $a_{m}, b_{n}$ in $A$ and $\phi$ in $A^{\prime}$, the two repeated limits of $\phi\left(a_{m} b_{n}\right)$ exist and differ.

Proposition 1. Ea $[-2 \pi, 2 \pi]$ is Arens irregular.

Proof. Let $0<\beta<1$ and define, for $z \in \mathbb{C}$,

$$
f(z)=\pi^{2} /\left[\Gamma(z)^{2} \Gamma(1+\beta-z) \Gamma(1-\beta-z)\right] .
$$

Since $1 / \Gamma$ is entire, so is $f$. By $\left[3\right.$, p. 47(5)], for $\operatorname{Re} z>0$ and $\alpha \in \mathbb{C}, z^{\alpha} \Gamma(z) / \Gamma(z+\alpha) \rightarrow 1$ uniformly as $|z| \rightarrow \infty$. Hence $\Gamma(z-\beta) \Gamma(z+\beta) / \Gamma(z)^{2} \rightarrow 1$, and so we obtain here $f(z) /[\sin \pi(z-\beta) \sin \pi(z+\beta)] \rightarrow 1$ as $|z| \rightarrow \infty, \operatorname{Re} z>0$. For $\operatorname{Re} z<0$ we find similarly that $f(z) / \sin ^{2} \pi z \rightarrow 1$ as $|z| \rightarrow \infty$. These imply that $f$ satisfies the above mentioned conditions so that $\phi\left(e^{i z h}\right)=f(z)$ defines $\phi \in E a[-2 \pi, 2 \pi]^{\prime}$.

Take $a_{m}=e^{i m h}$ and $b_{n}=e^{-i n h}(m, n \in \mathbb{N})$, so that $\left\|a_{m}\right\|=\left\|b_{n}\right\|=1$. Then $\phi\left(a_{m} b_{n}\right)=$ $f(m-n)$. The limits involving $f(z)$ give $\lim _{k \rightarrow \infty, k \in \mathbb{Z}} f(k)=-\sin ^{2} \pi \beta \neq 0=\lim _{k \rightarrow-\infty, k \in \mathbb{Z}} f(k)$. Hence by Pym's criterion $E a[-2 \pi, 2 \pi]$ is Arens irregular.

Turning to the inequalities, we rephrase theorem 3 of [4] equivalently as follows.

Proposition 2. If all the zeros $z_{k}$ of $p(z)=\alpha z^{2}+\beta z+\gamma$ have $\operatorname{Im} z_{k} \leqslant 0$, then for $f \in B_{\mathrm{r}}$,

$$
\left|\alpha f^{\prime \prime}(x)+\beta f^{\prime}(x)+\gamma f(x)\right| \leqslant|p(i \tau)| \sup \{|f(t)|: t \in \mathbb{R}\} \quad(x \in \mathbb{R}) .
$$

Proof. Since the operator $i D$ is Hermitian, with $\operatorname{Sp}(D)=[-\tau i, \tau i],[6]$ gives that for any $\zeta \in \mathbb{C}, D-\zeta I$ has norm equal to spectral radius, and so $\|D-\zeta I\|=\max \{\mid \zeta+$ $\tau i|,| \zeta-\tau i \mid\}$. Hence $\left\|D-z_{k} I\right\|=\left|\tau i-z_{k}\right|$ for each $k$, and so $\|p(D)\| \leqslant|p(\tau i)|$. Since $p(\tau i) \in \mathrm{Sp}(p(D))$ we have $\|p(D)\|-|p(\tau i)|$, which is the required result. 
This argument shows that the proposition holds for polynomials of any degree. The inequality of theorem 4 of [4] follows similarly: we restrict $D$ to the subspace of $B_{\tau}$ of functions $f$ with $|f(z)|$ bounded for $\operatorname{Im} z \geqslant 0$, which gives now $\operatorname{Sp}(D)=[0, \tau i]$.

The Arens regularity problem when $K$ has interior remains open. We are grateful to $J$. Duncan for useful discussions on this topic.

\section{REFERENCES}

1. M. J. Crabb, J. Duncan and C. M. McGregor, Some extremal problems in the theory of numerical ranges, Acta Math. 128 (1972), 123-42.

2. M. J. Crabb and C. M. McGregor, Polynomials in a Hermitian element, Glasgow Math. J. 30 (1988), 171-6.

3. A. Erdelyi, Higher transcendental functions, Vol. 1 (McGraw-Hill, 1953).

4. C. Frappier, Inequalities for entire functions of exponential type, Canad. Math. Bull. 27 (1984) 463-71.

5. J. S. Pym, The convolution of functionals on spaces of bounded functions, Proc. London Math. Soc. (3) 15 (1965) 84-104.

6. A. M. Sinclair, The norm of a Hermitian element in a Banach algebra, Proc. Amer. Math. Soc. 28 (1971) 446-50.

Department of Mathematics

UNIVERSITY OF GLASGOW

GLASGOW G12 8QW 